\title{
A large area timing RPC prototype for ion collisions in the HADES spectrometer
}

\author{
H. Alvarez Pol ${ }^{\mathrm{a}}$, R. Alves ${ }^{\mathrm{b}}$, A. Blanco ${ }^{\mathrm{b}}, \mathrm{N}$. Carolino $^{\mathrm{b}}, \mathrm{J}_{\text {. Eschke }}^{\mathrm{c}}$, \\ R. Ferreira-Marques ${ }^{\mathrm{b}, \mathrm{d}}$, P. Fonte ${ }^{\mathrm{b}, \mathrm{e}}$, J.A. Garzón ${ }^{\mathrm{a}}$, D. González Díaz ${ }^{\mathrm{a}, *}$,

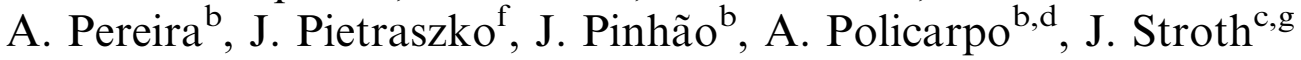 \\ ${ }^{\text {a }}$ Facutad de Fisica, Universidade de Santiago de Compostela, Campus sur, Santiago de Compostela, Spain \\ ${ }^{\mathrm{b}}$ LIP, Coimbra, Portugal \\ ${ }^{\mathrm{c}}$ GSI, Darmstadt, Germany \\ ${ }^{\mathrm{d}}$ Universidade de Coimbra, Coimbra, Portugal \\ ' ISEC, Coimbra, Portugal \\ ${ }^{\mathrm{f}}$ Smoluchowski Institute of Physics, Cracow, Poland \\ ${ }^{\mathrm{g}}$ Johann Wolfgang Goethe-Universität, Frankfurt, Germany \\ For the HADES collaboration \\ Available online 1 September 2004
}

\begin{abstract}
We present a resistive plate chamber (RPC) prototype for time-of-flight measurements over large areas and at high occupancies, minimizing the inter-channel cross-talk.

A procedure for the stand-alone calibration of the detector using redundant information is proposed, taking advantage of the very good spatial uniformity observed.

Measurements were performed at the GSI (Darmstadt) SIS accelerator for primary collisions of $\mathrm{C}$ at $1 \mathrm{GeV} / u$, as a first step towards the projected high acceptance di-electron spectrometer (HADES) upgrade to work at the highest multiplicities expected in $\mathrm{Au}-\mathrm{Au}$ collisions.
\end{abstract}

(C) 2004 Published by Elsevier B.V.

PACS: $29.40 . \mathrm{Cs}$

Keywords: RPC; TOF; Timing; Cross-talk; Ion collisions; HADES

*Corresponding author. Tel.: + 34-659-124-332.

E-mail address: diego@fpddux.usc.es (D. González Díaz).

\section{The HADES spectrometer}

The HADES (high acceptance di-electron Spectrometer) experiment, located at the GSI institute 
in Darmstadt, Germany, has been designed to improve the understanding of nuclear matter at high pressures. Its main goal is the study of the inmedium effects on the vector mesons, through the measurements of their decay into lepton pairs.

The physics program has already started with $\mathrm{C}-\mathrm{C}$ collisions at $1-2 \mathrm{GeV} / u$ and $\mathrm{Au}-\mathrm{Au}$ collisions at $1-1.5 \mathrm{GeV} / u$ are expected in the near future. To deal with the higher expected multiplicities, a new inner TOF detector for trigger levels 1 (centrality) and 2 (lepton candidate) is needed. Regarding particle identification (PID) capability, the detector must have a time resolution better than $100 \mathrm{ps} \sigma[1]$ and it must be able to work at SIS intensities of $2 \times 10^{7} \mathrm{~Hz}$, implying rate densities up to $700 \mathrm{~Hz} / \mathrm{cm}^{2}$ in the case of $\mathrm{Au}-\mathrm{Au}$ at $1.5 \mathrm{GeV} / u$. Timing resistive plate chambers (RPCs) offer a very promising solution, providing a sufficient time resolution at the required counting rate and affordable cost (Fig. 1).

\section{Timing RPCs}

RPCs are a 23-year-old technology [2], but only 4 years ago was it shown that it is possible to use
RPCs for precise time-of-flight measurements at normal conditions of pressure and temperature with inexpensive materials [3]. There are already several experiments foreseeing or working with timing RPCs such as ALICE [4], FOPI [5], HARP [6] or STAR [7].

\subsection{Cross-talk in high-occupancy environments}

Simulation indicates that the occupancy per cell should be at the level of $20 \%$ in the most unfavourable scenario. Roughly speaking, once there is a hit in a given cell, in a fraction $P_{\text {occ }}$ of the cases there is a second hit in the same cell and also in at least one of the neighbours with probability $N_{\text {neighbours }} \times P_{\text {occ. }}$. If these 'neighbour hits' interfere with the 'interesting' hit (cross-talk) the performance of the detector may deteriorate for a large fraction of the hits, potentially introducing complex systematic effects on the measurements.

\subsection{Shielded RPCs}

To avoid potentially troublesome cross-talk we have proposed and tested the shielded RPC layout
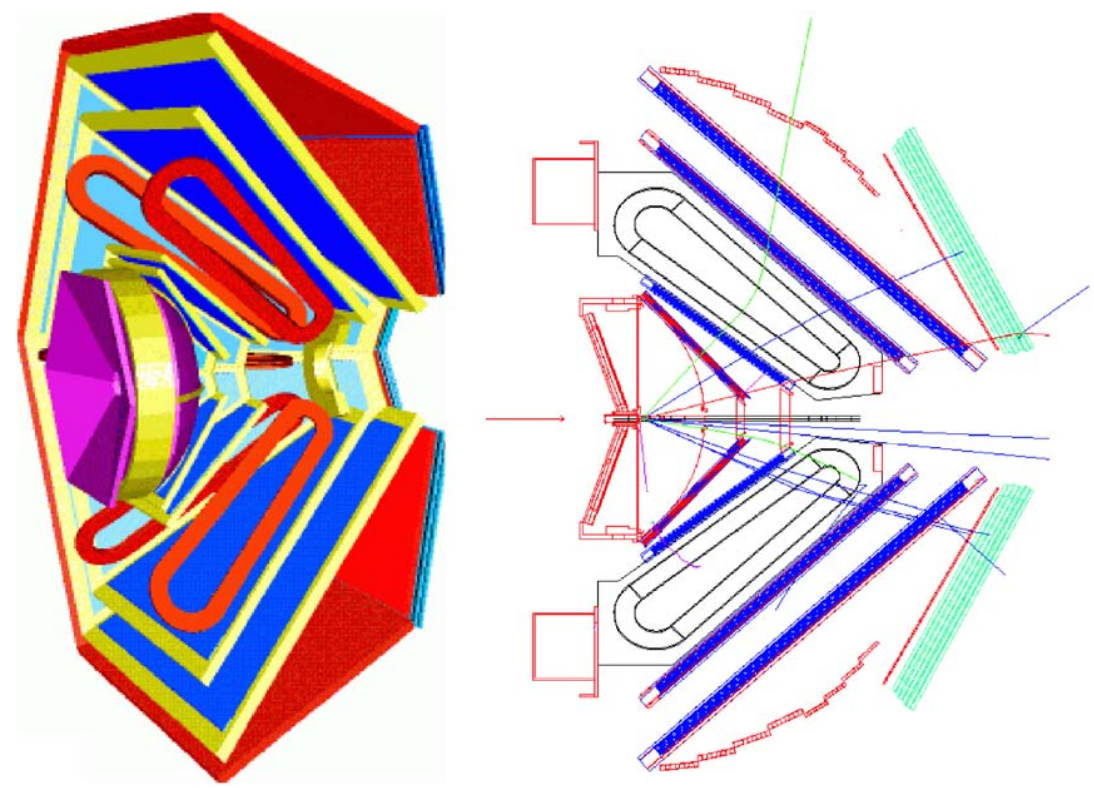

Fig. 1. HADES schematic views showing from left to right the RICH, inner MDCs, toroidal magnet, outer MDCs, TOF wall and the calorimeter (SHOWER). 


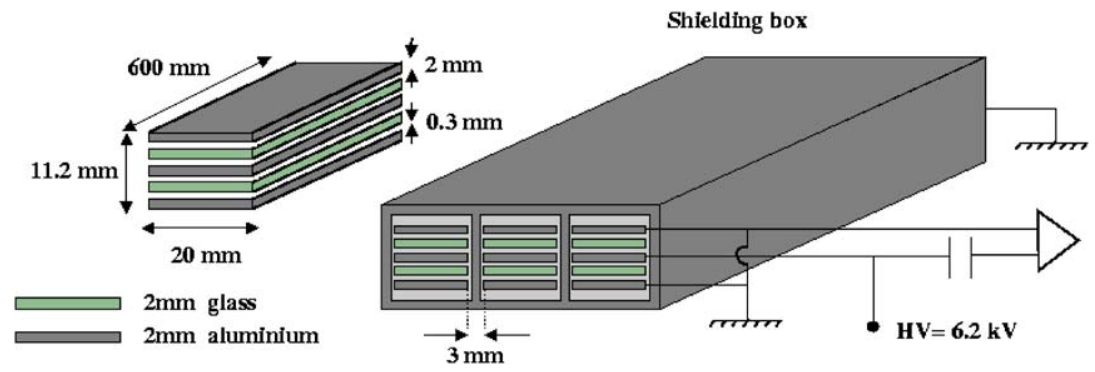

Fig. 2. Schematic representation of the RPC prototype.

shown in Fig. 2, resembling the original idea suggested in Ref. [9]. The chambers are constituted by $2 \times 60 \mathrm{~cm}^{2}$ large, $2 \mathrm{~mm}$ thick aluminium and glass electrodes, the latter being left electrically floating [10] for easier construction. Each RPC cell is shielded in grounded aluminium boxes (for details see Ref. [11]).

\section{Measurements}

\subsection{Physical environment}

We took data at the GSI SIS facility for primary interactions of $\mathrm{C}$ ions at $1 \mathrm{GeV} / u$. For the reference time, we used a scintillator system of resolution equal to $25 \mathrm{ps} \sigma$, which allowed to precisely measure the intrinsic resolution of the RPC and also the $\gamma \beta$ distribution of the incoming particles, yielding the energy deposition distribution. It was estimated that at least $5 \%$ of the particles deposited at least by a factor of 2 more energy than minimum ionizing particles [11].

\subsection{Cross-talk}

Despite previous experience of cross-talk levels around $85 \%$ between neighbouring strips for long detectors [8], we observed an excess of events of only $0.4 \%$ /neighbour due to the adequate shielding. As expected, the probability that a signal induces cross-talk is higher for larger signals (mostly from streamer discharges).

However, the definitive test is to measure the possible degradation of the time resolution performance in the case where there are two hits
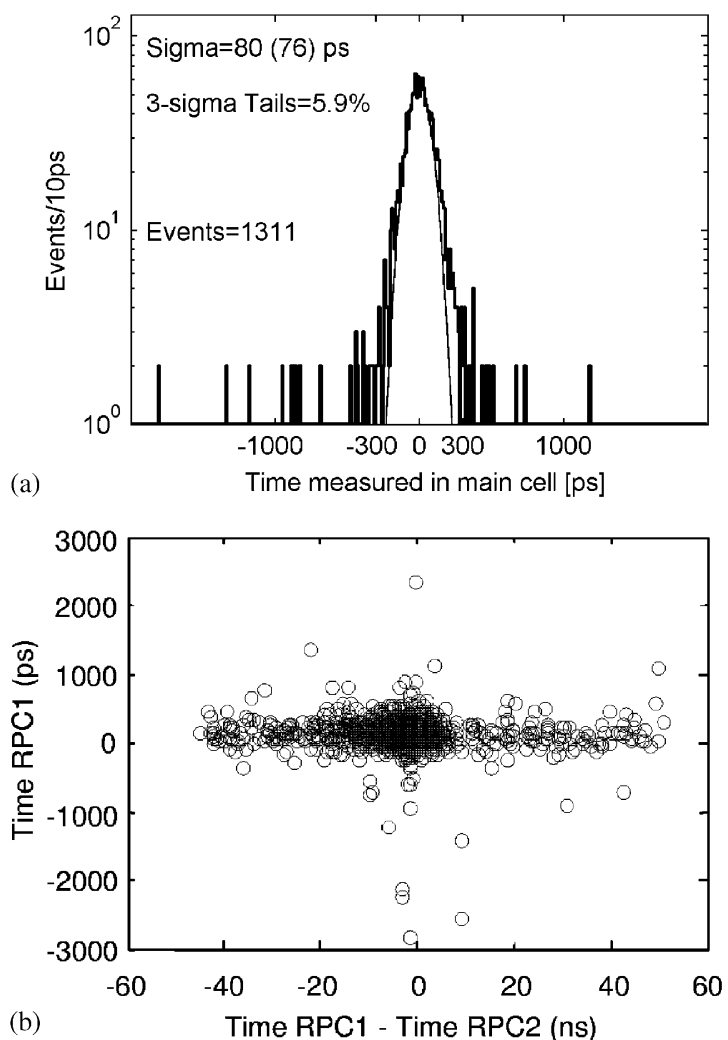

Fig. 3. (a) Time distribution in one RPC in presence of an almost simultaneous hit in a neighbouring cell. (b) No correlation between the time measured in the main counter and the time lag between both hits is observed.

almost simultaneously in neighbouring cells. Due to the low multiplicity of carbon collisions the probability of having 'neighbouring hits' was only $1.2 \% /$ neighbour. However, a sample of around 1300 of such events was collected with the 
corresponding unbiased time distribution shown in Fig. 3. It is also shown that there is no correlation between the time difference between both hits and the time measured in the 'interesting' channel.

\subsection{Rate behaviour}

The behaviour of some relevant observables is shown in Fig. $4: \varepsilon=87-\frac{1.5}{100 \mathrm{~Hz} / \mathrm{cm}^{2}}[\%], \sigma_{t}=67+$ $\frac{4.7}{100 \mathrm{~Hz} / \mathrm{cm}^{2}}[\mathrm{ps}]$ and $3 \sigma$ tails $=6.7+\frac{0.6}{100 \mathrm{~Hz} / \mathrm{cm}^{2}}[\%]$ ( $3 \sigma$ tails stands for the fraction of events outside $3 \sigma$ from the maximum).

RPCs similar to the ones used are known to yield efficiencies up to $99.5 \%$ [12], suggesting that the measured efficiency was dominated by geometric losses. The observed intrinsic inefficiency of $13 \%$ is compatible with the $15 \%$ expected for perpendicular particle incidence $(1-\varepsilon=$ dead zone/active zone $\simeq 3 \mathrm{~mm} / 20 \mathrm{~mm} \simeq 15 \%$ ). This effect suggests the adoption of a 2-layer scheme, which would provide the extra benefit of easier mechanical construction and redundant information useful for calibration purposes.

The use of thinner or slightly less resistive glass would further extend the counting rate capability and will be investigated in the future.

\subsection{Homogeneity and calibration}

The detector has shown good homogeneity along the longitudinal dimension (Fig. 5). The position resolution was measured to be below $6 \mathrm{~mm} \sigma$, with systematic shifts of the distribution below $2.5 \mathrm{~mm}$. These facts demonstrate the mechanical robustness of the design and the negligible effect of the nylon monofilaments used as spacers [11].

In timing RPCs the measured time must be corrected for the time-charge correlation that is always present [8]. Additionally, in any system, all timing signals must be guaranteed to be isochronous by adequate comparisons among themselves. All these procedures together are called calibration. In practice, this operation must be performed with some periodicity since the detector-related time lags most likely depend on environmental conditions.

In the present case it was found that:

(1) The time-charge correlation is well described by a simple two-segment linear fit in time vs. $\log (Q)$ representation. This can be particularly interesting for on-line purposes.

(2) The parameters of the fit can be obtained from any single position along the detector and used elsewhere.

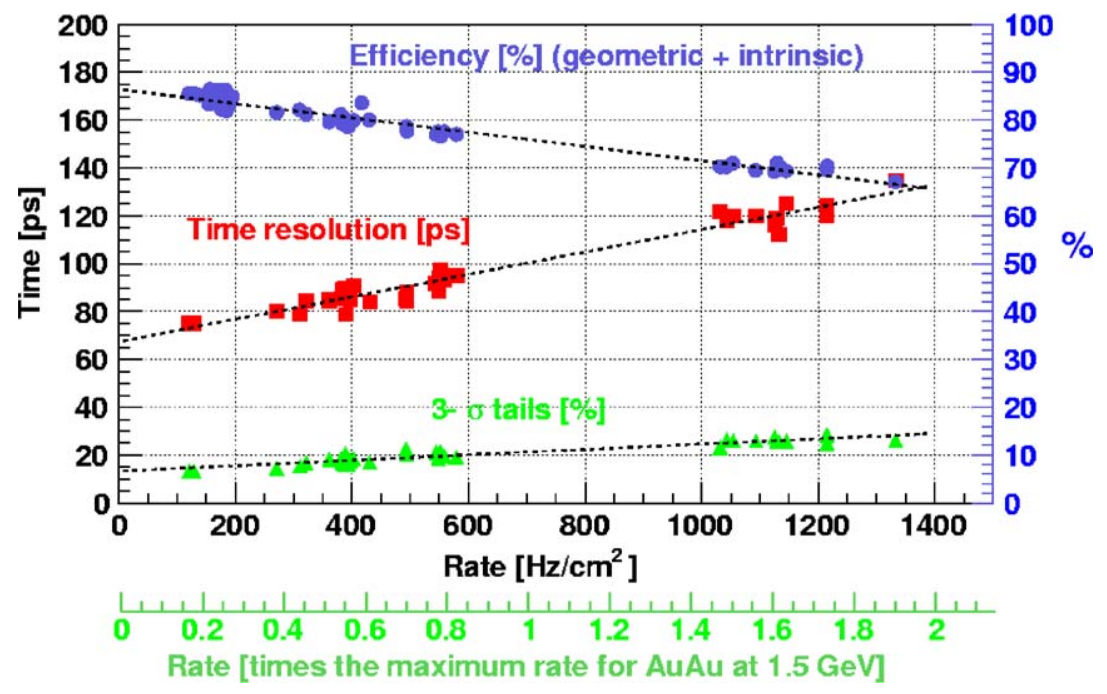

Fig. 4. Plot showing the global behaviour with rate of the efficiency (right axis), time resolution (left axis) and $3 \sigma$ tails (right axis). The equivalent expected maximum rates of operation at the HADES experiment are also shown. 


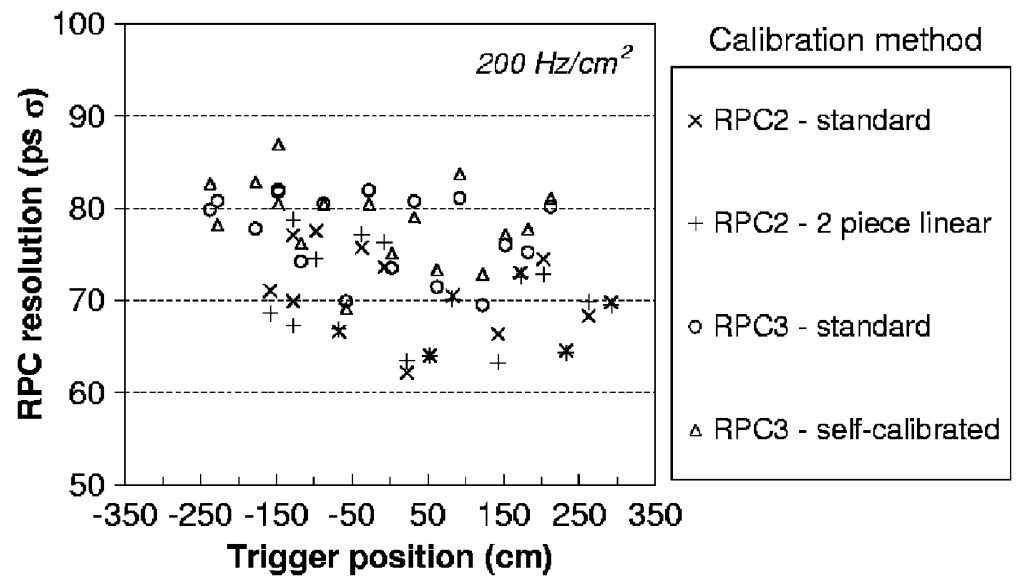

Fig. 5. Comparison between different calibration methods in several points along the detector. Standard: run-by-run fit in 15 linear segments. 2 piece linear: run-by-run fit in 2 linear segments. Self-calibrated: 2 piece linear fit derived by self-calibration (see Section 3.4.1) from a point chosen arbitrarily.

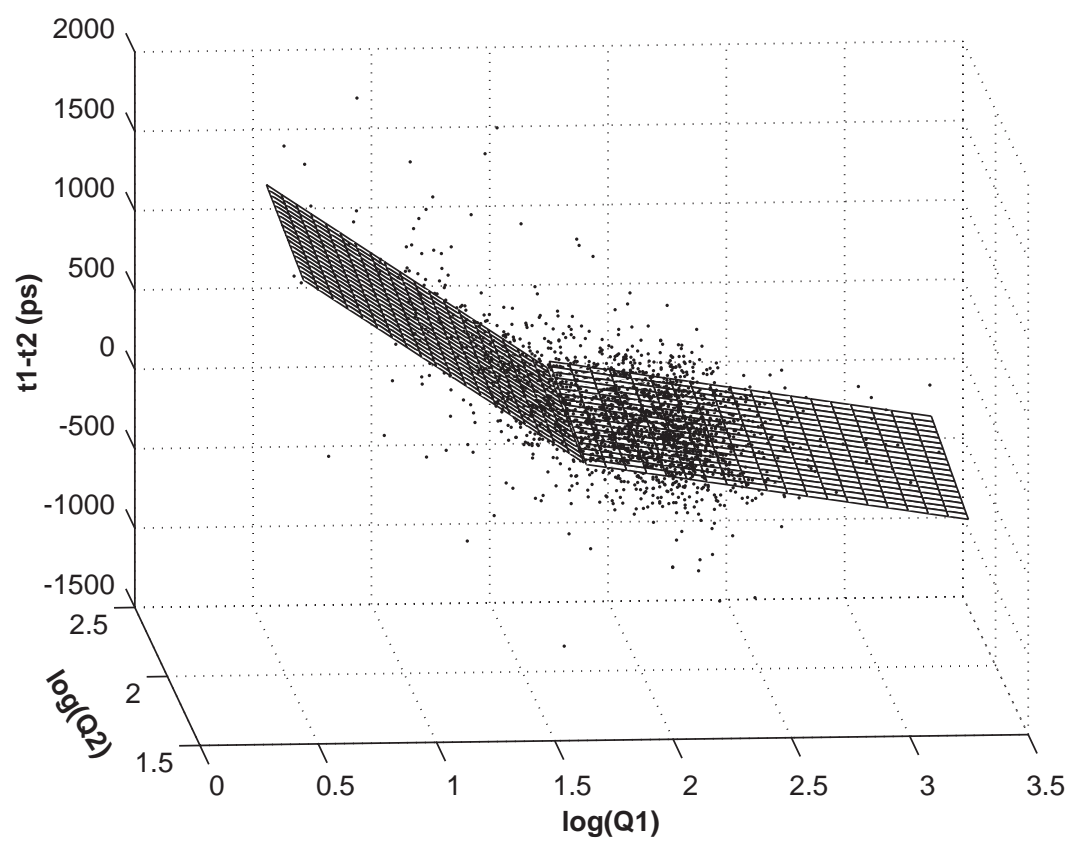

Fig. 6. Example of a self-calibration fit in two bilinear patches. The reference chamber corresponds to $Q_{2}$ and the fit parameters are calculated for chamber 1 (see Section 3.4.1).

(3) The time measured with two independent RPCs can be used to determine the parameters of the fit without the help of any auxiliary detector; we call this concept self-calibration and describe it in Section 3.4.1.

\subsubsection{Self-calibration}

The time difference between two independent RPCs placed close to each other and measuring the same particles is a function of both RPC charges only (Fig. 6). To derive the time-charge calibration for $\mathrm{RPC}_{1}$, for instance, we keep only a 
narrow range of $Q_{2}$ close to the charge distribution maximum and fit the points $\Delta t\left(Q_{1}, Q_{2}\right)$ by a mosaic of two bilinear patches, yielding the required linear correction segments for $\mathrm{RPC}_{1}$.

For the data shown in Fig. 5 the second RPC was simulated by data taken from an independent run at a single location chosen arbitrarily.

\section{Conclusion}

We have presented a shielded timing RPC design for large areas, with time resolutions of the order of $75 \mathrm{ps} \sigma$, good uniformity and negligible cross-talk.

The performance parameters change with the counting rate in the following way: $\varepsilon=$ $87-\frac{1.5}{100 \mathrm{~Hz} / \mathrm{cm}^{2}}[\%], \sigma_{t}=67+\frac{4.7}{100 \mathrm{~Hz} / \mathrm{cm}^{2}}[p s]$ and $3 \sigma$ tails $=6.7+\frac{0.6}{100 \mathrm{~Hz} / \mathrm{cm}^{2}}[\%]$, all remaining within the HADES requirements. The rate capability may be improved by using thinner or lower resistivity glass electrodes.

In order to meet the problem of the geometric inefficiency arising from the needed shielding space we plan to study a geometry in 2 layers, allowing for easier construction, redundancy and the possibility of self-calibration of the system.

The test was made under the conditions expected for ion collisions at $1-2 \mathrm{GeV} / u$, showing that an admixture of a few percent of highly ionising, low-energy particles does not significantly affect the performance of the timing RPC detector.

\section{Acknowledgements}

This work was co-financed by Fundacao para a Ciencia e Tecnologia project POCTI/FNU/49513/ 2002 and FEDER, MCYT FPA2000-2041-C02-02, FPA2003-7581-C02-02, XUGA PGIDT-02-PXIC20605-PN. The authors gratefully acknowledge C. Soares from VITRAN-Sociedade Transformadora de Vidros Lda, for his help in the construction of the detector.

\section{References}

[1] HADES, Technical proposal, GSI 1994.

[2] R. Santonico, R. Cardarelli, Nucl. Instr. and Meth. A 187 (1981) 377.

[3] P. Fonte, et al., Nucl. Instr. and Meth. A 443 (2000) 201.

[4] R. Arnaldi, et al., Nucl. Instr. and Meth. A 451 (2000) 462.

[5] A. Schuttauf, VII RPC workshop, 2003.

[6] M. Bogomilov, et al., Nucl. Instr. and Meth. A 508 (2003) 152.

[7] F. Geurts, VII RPC workshop, 2003.

[8] A. Blanco, et al., Nucl. Instr. and Meth. A 485 (2002) 328.

[9] Ch. Finck, et al., Nucl. Instr. and Meth. A 508 (2003) 63.

[10] E. Cerron Zeballos, et al., Nucl. Instr. and Meth. A 374 (1996) 132.

[11] H. Alvarez-Pol, et al., VII RPC workshop, 2003.

[12] P. Fonte, et al., Nucl. Instr. and Meth. A 49 (2000) 295. 